\title{
Cloud-Based Automated Power Factor Correction and Power Monitoring
}

\author{
Mafaz M. Shubbar*, Laith A. Abdul-Rahaim, Ahmed A. Hamad \\ Electrical Engineering Department, College of Engineering, University of Babylon, Hilla, Babil 51001, Iraq
}

Corresponding Author Email: mafaz.abed.engh342@student.uobabylon.edu.iq

https://doi.org/10.18280/mmep.080510

Received: 16 June 2021

Accepted: 10 August 2021

\section{Keywords:}

cloud computing, power factor correction, APFC, IoT, neural networks, NodeMCU, Wi-Fi, computational

\begin{abstract}
Energetic life-sustaining needs, such as electrical power, are essential for everyday existence. It is commonly used in residential, industrial, farming, and medical facilities. Life without energy is minimal. Despite the vital need for electricity demand, losses curtailments and additional energy bills are still problems. Power factor correction is a method to fix or minimize mentioned problems. Automated power factor correction (APFC) will precede good contrivance for correction. Several studies on established systems endeavoured to improve power factor via local calculation and correction, android application, or web monitoring with disparity results and node types. The purpose of this treatise is to suggest a neoteric cloud APFC with neural network design advances to recent designs of APFC that depend on IoT and cloud. This design used a private cloud utilizing raspberry pi and a neural network to correct the power factor of homes in a single algorithm, and cloud helping in hosting and accessed on-demand at any time and from everywhere as long as the Internet is accessible and the neural for determining the capacitance value for power factor correction. In addition, this design will minimize devices used, give precise results, minimize the cost of the bill and make the easy utility monitoring of the power factor before and after correction.
\end{abstract}

\section{INTRODUCTION}

In the past few years, the change from offline procedures to online ones occurs in the power systems. An online technique employs smart and resilient sensors to pick up real-time data, but we need a platform that runs around the clock to store, evaluate, and reply. Cloud computing can fulfil the previous requirement [1]. In the first pivot, cloud the service provided to customers by cloud computing, which may offer different capabilities like hardware, databases, storage, networks, and software applications. When utilized, abundant financial benefits are available for the organization, people, and ventures, while showcasing remarkable cooperation opportunities [1, 2]. Cloud computing is one of the most innovative IT fields; it has been made public for a while and will be in use for years to come [2]. Cloud service provides dependability, responsiveness, broad ingress for network, position-dependent placement rules, flexibility, voluntary applications, distribution, scalability, dynamism, performance-enhanced services modification, finite expenditures, and unification of resources across many application domains $[3,4]$. Depending on the demand, there are four kinds of cloud computing models Public, particular owned (Private), mixed between mentioned two models named Hybrid and district or Community [5], as shown in Figure 1. In addition, there are three different sorts of cloud computing offerings which are referred to as follows $[1,5]$ :

1. IaaS (Infrastructure as a Service);

2. PaaS (Platform as a Service);

3. SaaS (Software as a Service).

The first service granted client of the cloud the aptitude to access virtual computing resources on demand. In IaaS, items of IT infrastructure like servers, networking, and storage are managed and virtualized. At the same time, the second one (PaaS) proffers an alternative to working virtual infrastructure components, using software development and hosting peripherals and can be utilized from the third service (SaaS) in web-based applications [3].

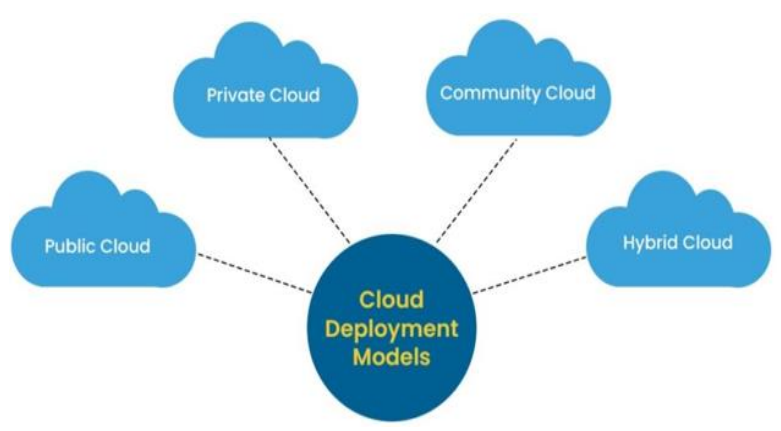

Figure 1. Cloud computing types

Cloud computing utilities are built web-based, maintained, and paid for effortlessly after being introduced, with development, administration, and payment made through the web [6]. On the other hand, using cloud computing trots out significant advantages for many entities, including healthcare organizations that convey care services and commodities and home automation, and innovative home systems [7]. As a result, a large and valuable amount of data is available linked devices and appliances in the intelligent residences managed by an IoT system [3]. The analysis process on this data in semi real-time can give a diverse assortment of information that carries enormous management and operational costs [8]. Also, 
online response for cloud systems allows vendors to scrutinize data regularly and fix any equipment that suffers from impairment meet or damage [9]. The second axis is the power factor which is exemplified as a voltage-versus-current phase shift in AC systems. Reactive power and power factor can be affected by whether the system contains a capacitive or inductive load. Consistently, the power factor may be lead or lag contingent on accentuating the capacitive or inductive load. On the other hand, an inductive load proposes a lagging power factor due to the retardation current, which may be recouped by switching the relevant capacitance to the system.

In contrast, a capacitive load suggests a leading power factor, which may be repaid by introducing equivalent inductance [10]. However, most electric loads have inductive nature, which means they produce a lagging power factor. This is detrimental, as it creates a rise in current flow and eventuates in extra loss of the active power; hence it escalates the overall loss of power in the system [11]. Due to the above reason and it pertains to the economy, maintaining the power factor as near to unity as possible is crucial. However, as most power system parts require a constant quantity of reactive power to function, it is virtually impossible [12]. The final basis subject is power factor improvement which is the process to offset the unwanted effects on the different electric loads when the system meets a low power factor by providing the reactive power necessary for roughly improving the power factor to unity, and this is generally accomplished by adding capacitors to the electrical network with no influence on the equipment's functionality [10]. Using the proper power factor adjustment has these advantages [13]:

a- Power consumption is reduced through greater energy efficiency, which translates into environmental gain. Less demand equals less greenhouse gas emissions and fossil fuel depletion from power plants reducing monthly electric bills.

b- It plays a role in the minimization of iron distribution equipment losses the voltage drop.

c- Overall electrical equipment and components' life is extended.

The purpose of this paper is to suggest a novel design of home power factor correction automatically based on cloud computing (PaaS) and neural network after auditing current methodologies for automatic power factor correction with IoT and cloud. The neural network is employed to predict the capacitance values that compensate for the reactive power to correct the power factor. The Raspberry Pi works as a private cloud central unit to perform and run the neural network algorithm to correct all homes power factors in one algorithm. This method gives prices results because it depends on neural networks.

This design surpasses the methods mentioned later in the literature survey. It supports multi-home correction in one central device, so this will decrease the used number of devices. In contrast, previous methods used separated processes for each home power factor correction. Further, this system can be extended to manage big data or big district power quality enhancement via power factor correction. Also, it emphasizes the micro or mini-grid principle. Our core design allows monitoring before and after the correction process of the power factor.

This article is marshaled as follows. Section 2 shows the previous automatic power factor correction methods based on IoT and cloud. In section 3 states the shortcomings of anterior studies. Section 4 represents the proposed architecture of power factor correction based on cloud computing and neural networks. While in section 5, the features of the proposed system were exposed over the previously studied systems. In section 6 , the steps of the power factor correction process were shown. In addition, section 7 stated the tools that must be used to implement the system. Finally, in section 8 , the illation of this work was exhibited.

\section{LITERATURE SURVEY}

Gunawan et al. [11] found during experiments on the calibration of the current measurement in the power factor meter that the accuracy increased utilizing an SD card to store the detected data points and send them to MATLAB's user interface for monitoring. In addition, smart meter IoT framework analysis was covered. Automatic power factor adjustment was made through parallel linked capacitors; Arduino coupled to a relay circuit might control switches that activate the capacitors switch selector for capacitor bank with conducting trials on a wide range of electrical appliances and collecting data may improve automatic power factor correction. Taye [14] designed and emulated an automated power factor adjustment prototype utilizing an Arduino as a microcontroller. The usage of this kit decreased expenses and helped customers because the industry power factor was enhanced from 0.66 to 0.92 . Similarly, Vignesh Kumar et al. [13] constructed a scheme to reduce the penalty for industrial facilities, a set of automated power factor correction (APFC) equipment has been created around the Raspberry Pi. The industrial sector can benefit from the power factor adjustment technology - system and equipment efficiency both rises. For safety, attention should be paid to power factor adjustment. Otherwise, the voltage and current will increase, and power system or machine components will be more likely to break, and capacitor bank life would be shortened. Furthermore, Sathiyapriya et al. [15] studied several induction motor parameters and continually updated these data into a site utilizing IoT. Alarm messages were sent to the individual who needs to know, and an electrical relay activated. This system used a changing load to simulate the energy consumption of an induction motor and found out whether or not it is possible to automatically enhance the motor's power factor. The benefit of reduced power factor penalty and the utilities' protection of induction motors are described. Microcontroller and capacitor banks were used to develop power factor correction equipment that boosted by 0.21 from 0.76 when under test load settings. About $1.7 \%$ of the planned load was saved in energy use. On the other hand, Thamizharasan et al. [16] wielded the signal's on-time status to determine the power factor value when the voltage and current signals were in phase difference. The bank capacitors were used to increase power quality and efficiency through the power factor correction, and the requisite power factor capacitor value was computed using Programmable Interface Circuit (PIC) logically. Bhagavathy et al. [17] built an Android application; this app helped the user inspect the power factor revision and a bank capacitors misstep. The fluctuation effect of the power factor owing to the failure of the capacitor bank was categorized. When the power factor scale varies, the panel of APFC swaps capacitor banks automatically, restoring power factor unity, and power factor fluctuations were investigated under various load circumstances. Also, the changes in load can cause a rapid shift in power factor. Conversely, Praveen et al. [18] addressed 
domestic load monitoring, informed the proper power factor range of the concerned load and which appliance degrade the power factor. The simulation results were produced using the Proteus program and suggested that the load's inadequate power factor must be determined using the proposed technique. IoT was helping customers, and electric company employees better understand how their loads and power factors affected one another. Non-linear household loads were concerned; in addition, the consumer will be provided with information about the load generating low power factor and how to minimize it through IoT. Mohammed et al. [19] used a Wi-Fi kit named Photon to control and monitor the Mosul University electricity plant activities remotely. Supervise voltage, devices load current, and valuations power factor were included in this term. Additionally, the system was developed to use an automatic power factor adjustment approach, fire detection and alarm unit, and carbon monoxide detector. In a major accident, such as a fire, the plant's security system can lock down the facility to prevent more incidents. The equipment mentioned above was utilized to upload the sensor's data to the ThingSpeak cloud, to be processed fast and visualized. Alternatively, Tarase et al. [20] proposed a methodology for voltage and current monitoring system via monitoring a simple one-phase electric system by utilizing an Arduino microcontroller in reading sensors observed values of voltagecurrent over wireless transmitting the gathered information to a new Android application to monitor the results. The system estimated both the voltage and current and the frequency of line and power factor by employing detectors for zero crossings. A new Android smartphone application has been built using MIT App Inventor 2 to be used in the monitoring system. This program was capable of fetching data of 3- $\Phi$ RMS voltage and current via Bluetooth. Gomaa et al. [21] devoted a mathematical model tested on the cabling industry through a MATLAB model mason. While the IoT physical layer gathered, evaluated, and transmitted electric power characteristics associated with manufacturing process data obtained from PLC IoT nodes. Without the installation of extra correction nodes, the adequate power quality was improved through manufacturing process management, so $33 \%$ in the power factor from (0.7 to 0.93$)$ was achieved. The implemented model suggested a successful process management idea while minimizing the need for correction nodes while achieving the same production outcomes. Nugroho et al. [22] was used the Neural Network approach to automatically reckoning and rectifying power factor, and power may be monitored online via IoT. Overall, $97.8 \%$ of the appliance's load trained and $94.8 \%$ of the untrained ones benefited from using this power factor enhancement technology. Using the MQTT protocol in the observation process based on IoT improved data transfer efficiency, enabling real-time monitoring on the website. Table 1 below represents a comparison between systems according to specific characteristics.

Table 1. Literature review of automatic power factor approaches according to (hardware core, User Interface (UI), sensors or actuators, node type, power factor correction way and messaging protocol)

\begin{tabular}{|c|c|c|c|c|c|c|c|}
\hline Reference & $\begin{array}{c}\text { Core hardware } \\
\text { device }\end{array}$ & UI & $\begin{array}{c}\text { Actuators or } \\
\text { Sensors }\end{array}$ & $\begin{array}{c}\begin{array}{c}\text { Communication } \\
\text { scheme }\end{array} \\
\end{array}$ & Node type & $\begin{array}{l}\text { Pf Correction } \\
\text { Way } \\
\end{array}$ & $\begin{array}{c}\text { Messaging } \\
\text { Protocol } \\
\end{array}$ \\
\hline [11] & Arduino & LCD & $\mathrm{CT}, \mathrm{VT}$ & Wi-Fi & Arduino & computational & - \\
\hline [14] & Arduino & LCD & $\mathrm{CT}, \mathrm{VT}$ & - & local & computational & - \\
\hline [13] & $\begin{array}{l}\text { Raspberry Pi } \\
\text { board }\end{array}$ & $\mathrm{PC}$ & CT, VT & - & $\begin{array}{l}\text { Raspberry Pi } \\
\text { board }\end{array}$ & computational & - \\
\hline$[15]$ & Arduino & $\begin{array}{l}\text { Web } \\
\text { page }\end{array}$ & CT, VT & Wi-Fi & NodeMCU & computational & НTTP \\
\hline [16] & PIC-16F877A & LCD & $\mathrm{CT}, \mathrm{VT}$ & Wi-Fi & - & computational & - \\
\hline [17] & PIC-16F877A & $\begin{array}{l}\text { Mobile } \\
\text { app }\end{array}$ & CT, VT & Wi-Fi & Wi-Fi module & computational & TCP \\
\hline [18] & Arduino & $\begin{array}{l}\text { Mobile } \\
\text { app }\end{array}$ & $\mathrm{CT}, \mathrm{VT}$ & Wi-Fi & IoT module & computational & TCP \\
\hline [19] & HL-56S V1.0 & $\begin{array}{l}\text { Web } \\
\text { page }\end{array}$ & $\begin{array}{c}\text { ZMPT101B, SCT- } \\
013-030\end{array}$ & $\mathrm{Wi}-\mathrm{Fi}$ & Particle Photon & computational & HTTP \\
\hline$[20]$ & Arduino & $\begin{array}{l}\text { Mobile } \\
\text { app }\end{array}$ & CT, VT & Wi-Fi & Arduino & computational & $\mathrm{TCP}$ \\
\hline [21] & $\begin{array}{c}\text { central processing } \\
\text { server }\end{array}$ & $\mathrm{PC}$ & CT, VT & Wi-Fi\& Bluetooth & IoT beacons & computational & MQTT \\
\hline$[22]$ & Stm32 & web page & pzem-004t & Wi-Fi & Esp8266 & Neural network & MQTT \\
\hline
\end{tabular}

\section{CHALLENGES OF PRESENT SYSTEMS}

There are various flaws with current systems as below:

(1) Some systems did not address the correction and monitoring of each device or appliance $[11,20]$.

(2) The majority of schemes deal with industrial loads and employ static capacitance values $[15,17,19,21]$.

(3) The estimation of capacitance that was added to boost the power factor was not exact in some projects [13, 19].

(4) Certain studies did not employ real-time data to assure machine failure prediction and power quality data computation [14].

(5) Adding extra capacitance can cause an inaccurate power factor in some research studies $[18,19]$.

(6) In most endeavours, in order to correct the lagging power factor, solid-state relays are used $[11,15,16]$.

(7) Treat each household or industrial load individually (each home or industry needs an algorithm for correction power factor) [22].

\section{PROPOSED METHODOLOGY}

The main goal of the future system is to provide knowledge concerned with cost reduction, multi-home process assistance, and core device decision-making. The new automatic power 
factor correction will be constructed to increase the monitoring and power quality benefits. Following the advances in electrical networks, our solution will be widely agreed upon and will have less cost for the user and need less reactive power. It leverages the private cloud idea as PaaS and may be expanded to accommodate hybrid cloud scenarios. A pretest will be run in the system to identify combinations of common devices to all homes in nonequal houses residential area according to the size of the home (big, middle, small). In the primary typical process device and made appropriate choices during the creation of training data (actual power, and power factor) for the neural network algorithm, then offered each house the best option for improving power factor by picking the perfect value of capacitance based on the algorithm conclusion. The block diagram for two homes is shown in Figure 2 below.

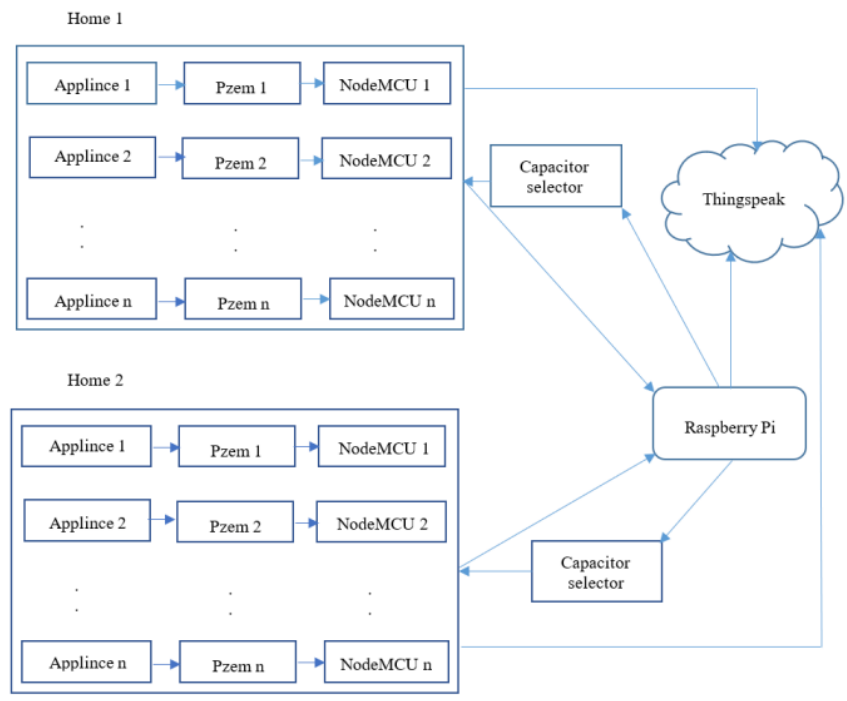

Figure 2. Block diagram design for two homes

\section{ADVANTAGES OF PROPOSED SYSTEM OVER EXISTING SYSTEM}

This proposed system surpasses the previous methods as shown in Table 2 below.

Table 2. Comparability of prospective system with other existent ones

\begin{tabular}{|c|c|}
\hline $\begin{array}{l}\text { New system } \\
\end{array}$ & Previous systems \\
\hline $\begin{array}{l}\text { Multi home correction in one } \\
\text { device }\end{array}$ & Separated correction algorithm \\
\hline $\begin{array}{l}\text { Scaling support and cloud } \\
\text { concept employ }\end{array}$ & $\begin{array}{l}\text { Major depend on IoT and less } \\
\text { focus on cloud }\end{array}$ \\
\hline $\begin{array}{l}\text { Precise power factor } \\
\text { correction }\end{array}$ & $\begin{array}{c}\text { Average power factor } \\
\text { correction }\end{array}$ \\
\hline Neural network function & Calculation based correction \\
\hline $\begin{array}{l}\text { Monitoring power and } \\
\text { correction process }\end{array}$ & $\begin{array}{l}\text { Some monitoring and other } \\
\text { only correction }\end{array}$ \\
\hline It simplified devices and cost & $\begin{array}{c}\text { Each unit has device and more } \\
\text { cost }\end{array}$ \\
\hline $\begin{array}{l}\text { Central mini or nano grid } \\
\text { support }\end{array}$ & Individual unit correction \\
\hline
\end{tabular}

\section{ALGORITHM OF PROPOSED SYSTEM}

- Measure the voltage, actual power, and power factor of a sample of household appliances by Pzem.

- Transmit the measured data to the Raspberry Pi via NodeMCU.

- $\quad$ Send power factor data to a web page (Thingspeak).

- Use a neural network to compute the appropriate capacitance for APFC.

- Save capacitance value in Raspberry Pi.

- Send the previous amount of capacitance to the capacitor selector.

- Add the capacitor to the correct power factor.

- Send corrected power factor data to the web page (Thingspeak).

- The procedure is ended.

\section{REQUIREMENTS AND COMPONENTS THAT SHOULD BE USED IN THE PROJECT}

\subsection{The peripherals}

- Raspberry $\mathrm{Pi}$ is a private cloud server that decides the appropriate value of condensation.

- NodeMCU data-sending node unit.

- Pzem-004t sensor device that read power values parameters with current transformer (CT) and voltage transformer (VT).

\subsection{The essentials}

○ Raspbian operation system software for Raspberry Pi.

- C language for NodeMCU.

- Python programming language will be used for algorithms inside raspberry pi.

\subsection{Database}

SQL or SQLite database to save the actual collected values of power factor and the generated values after correction for each home and capacitance value for correction.

\section{CONCLUSIONS}

This work will present a detailed study on designing a prototype of automated and automatic power factor correction based on the cloud in consonance with the examination of both APFC based on the cloud and the IoT approaches. Even though just a few studies have concentrated on the cloud computing precept, applying cloud computing principles will lead to more rigorous computation and can be expanded to add more residential units. Using this future study will cut down on time required and deliver accurate results and performance. In the right conditions, neural networks will assist in fulfilling the listed mentioned results. The system facilitates scaling and can reduce customer bills. It is also different from previous methods in that it uses central monitoring for all power factor correction processes and can be accessed from any device at any time. In addition, this study will help the consumer and the utility or company of electricity in monitor the power factor correction. and knowing the devices which made low power factor.

- Begin the process. 


\section{REFERENCES}

[1] Raju, M.P., Laxmi, A.J. (2020). IoT based online load forecasting using machine learning algorithms. Procedia Computer Science, 171: 551-560. http://dx.doi.org/10.1016/j.procs.2020.04.059

[2] El-Sayed, H., Sankar, S., Prasad, M., Puthal, D., Gupta, A., Mohanty, M., Lin, C.T. (2017). Edge of things: The big picture on the integration of edge, IoT and the cloud in a distributed computing environment. IEEE Access, 6: 1706-1717. http://dx.doi.org/10.1109/ACCESS.2017.2780087

[3] Yassine, A., Singh, S., Hossain, M.S., Muhammad, G. (2019). IoT big data analytics for smart homes with fog and cloud computing. Future Generation Computer Systems, 91: 563-573. http://dx.doi.org/10.1016/j.future.2018.08.040

[4] Muralidharan, S., Song, G., Ko, H. (2019). Monitoring and managing IoT applications in smart cities using kubernetes. Cloud Computing, pp. 1-6.

[5] Goyal, S. (2014). Public vs private vs hybrid vs community-cloud computing: A critical review. International Journal of Computer Network and Information $\quad$ Security, 6(3): 20-29. http://dx.doi.org/10.5815/ijcnis.2014.03.03

[6] Sultan, N. (2014). Making use of cloud computing for healthcare provision: Opportunities and challenges. International Journal of Information Management, 34(2): 177-184. http://dx.doi.org/10.1016/j.ijinfomgt.2013.12.011

[7] Lin, Y.H. (2019). Novel smart home system architecture facilitated with distributed and embedded flexible edge analytics in demand-side management. International Transactions on Electrical Energy Systems, 29(6): 2014 2019. http://dx.doi.org/10.1002/2050-7038.12014

[8] Memon, A.A., Wang, C., Naeem, M.R., Aamir, M., Ayoob, M. (2014). Cloud government - a proposed solution to better serve the nation. In Proceedings of 2014 International Conference on Cloud Computing and Internet of Things, $\mathrm{pp}$. 39-44. http://dx.doi.org/10.1109/CCIOT.2014.7062502

[9] Korkmaz, I., Metin, S.K., Gurek, A., Gur, C., Gurakin, C., Akdeniz, M. (2015). A cloud based and Android supported scalable home automation system. Computers \& Electrical Engineering, 43: 112-128. http://dx.doi.org/10.1016/j.compeleceng.2014.11.010

[10] Ananthapadmanabhan, V.R., Tenison, I., Shanavas, T.N. (2018). Automated power factor improvement based on artificial neural networks. In 2018 International Conference on Intelligent Autonomous Systems (ICoIAS), pp. 170-174. http://dx.doi.org/10.1109/ICoIAS.2018.8494126

[11] Gunawan, T.S., Anuar, M.H., Kartiwi, M., Janin, Z. (2018). Development of power factor meter using Arduino. In 2018 IEEE 5th International Conference on Smart Instrumentation, Measurement and Application (ICSIMA), pp.

http://dx.doi.org/10.1109/ICSIMA.2018.8688750

[12] Gomaa, N.N., Youssef, K.Y., Abouelatta, M. (2019). An IoT-based energy efficient system for industrial sector. In 2019 15th International Computer Engineering Conference (ICENCO), pp. 132-137. http://dx.doi.org/10.1109/ICENCO48310.2019.9027440

[13] Kumar, M.V. Dharani, M., Pavithra, Y., Vedha Varshini,
S. (2018). Automatic power factor controller using raspberry PI. International Journal of Electrical, Electronics and Data Communication (IJEEDC)IJEEDC, 6: 42-44. http://iraj.doionline.org/dx/IJEEDCIRAJ-DOIONLINE-11942.

[14] Taye, A. (2018). Design and simulation of automatic power factor correction for industry application. International Journal of Engineering Technologies and Management Research, 5(2): 10-21. http://dx.doi.org/10.29121/ijetmr.v5.i2.2018.142

[15] Sathiyapriya, P., Dhanasooryaa, S., Gokulakrishnan, S. (2019). Power Factor Monitoring and Controlling for Industrial Load using IoT. International Journal of Advanced Research in Electrical, Electronics and Instrumentation Engineering, 8(3): 555 -559.

[16] Thamizharasan, R., Kathiresan. S., Nandhini, D. (2019). Automatic power factor correction and monitoring using IoT. International Journal of Engineering Science and Computing, 9(5): 22423-22424.

[17] Bhagavathy, P., Latha, R., Thamizhmaran, E. (2019). Development of IoT enabled smart APFC panel for industrial loads. In 2019 10th International Conference on Computing, Communication and Networking Technologies (ICCCNT), pp. 1-5. http://dx.doi.org/10.1109/ICCCNT45670.2019.8944899

[18] Praveen, A.A.A., Kumaran, M.M., Ali, A.N., Premkumar, K. (2020). Minimization of power factor penalty charges for non-linear domestic loads with IOT technology. In IOP Conference Series: Materials Science and $\quad$ Engineering, 937(1): 012011. https://doi.org/10.1088/1757899X/937/1/012011

[19] Mohammed, E.A., Al-Allaf, A.F., Altamer, B.R. (2020). IoT-Based monitoring and management power substation of the University of Mosul. In IOP Conference Series: Materials Science and Engineering, 928(2): 022061. 899X/928/2/022061

[20] Tarase, K.B., Panchade, V.M. (2020). Monitoring \& controlling of substation using IoT in distribution power grid. In 2020 5th International Conference on Devices, Circuits and Systems (ICDCS), pp. 66-70. http://dx.doi.org/10.1109/ICDCS48716.2020.243550

[21] Gomaa, N.N., Youssef, K.Y., Abouelatta, M. (2021). On design of IoT-based power quality oriented grids for industrial sector. Advances in Science, Technology and Engineering Systems Journal, 5(6): 1634-1642. https://dx.doi.org/10.25046/aj0506194

[22] Nugroho, D.C., Mayaratri, Y., Syai'in, M., Hasin, M.K., Rohiem, N.H., Putra, N.P.U., Soeprijanto, A. (2021). Household electricity network monitoring based on IoT with of automatic power factors improvement using neural network method. In IOP Conference Series: Materials Science and Engineering, 1010(1): 012045. https://doi.org/10.1088/1757-899X/1010/1/012045

\section{NOMENCLATURE}

$\begin{array}{ll}\text { SD } & \text { Secure Digital } \\ \text { GUI } & \text { Graphical User Interface } \\ \text { RMS } & \text { Root Mean Square } \\ \text { CT } & \text { Current Transformer } \\ \text { VT } & \text { Voltage Transformer } \\ \text { NodeMCU, } & \text { protype board }\end{array}$


Esp8266

HTTP

MQTT

TCP

LCD

PIC, HL-56S
Hypertext Transfer Protocol Message Queuing Telemetry Transport

Transmission Control Protocol

Liquid Crystal Display

Microcontrollers
V1.0, Stm32

PLC

Pzem

SQL or SQLite
Programmable Logic Controller Module for measuring AC power parameters

Structured Query Language needed to query Relative Database System 